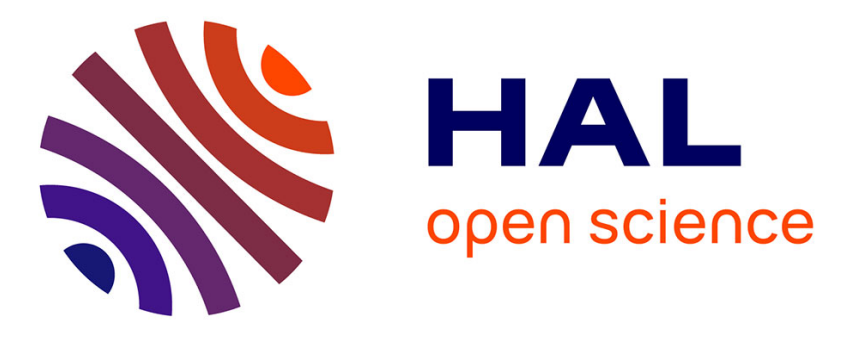

\title{
Exchange of avatars: Toward a better perception and understanding
}

Thomas Lopez, Rozenn Bouville Berthelot, Emilie Loup-Escande, Florian Nouviale, Valérie Gouranton, Bruno Arnaldi

\section{- To cite this version:}

Thomas Lopez, Rozenn Bouville Berthelot, Emilie Loup-Escande, Florian Nouviale, Valérie Gouranton, et al.. Exchange of avatars: Toward a better perception and understanding. IEEE Transactions on Visualization and Computer Graphics, 2014, 20 (4), pp.644-653. 10.1109/TVCG.2014.22 . hal01003200

\section{HAL Id: hal-01003200 \\ https://hal.science/hal-01003200}

Submitted on 10 Jun 2014

HAL is a multi-disciplinary open access archive for the deposit and dissemination of scientific research documents, whether they are published or not. The documents may come from teaching and research institutions in France or abroad, or from public or private research centers.
L'archive ouverte pluridisciplinaire HAL, est destinée au dépôt et à la diffusion de documents scientifiques de niveau recherche, publiés ou non, émanant des établissements d'enseignement et de recherche français ou étrangers, des laboratoires publics ou privés. 


\title{
Exchange of Avatars: Toward a Better Perception and Understanding
}

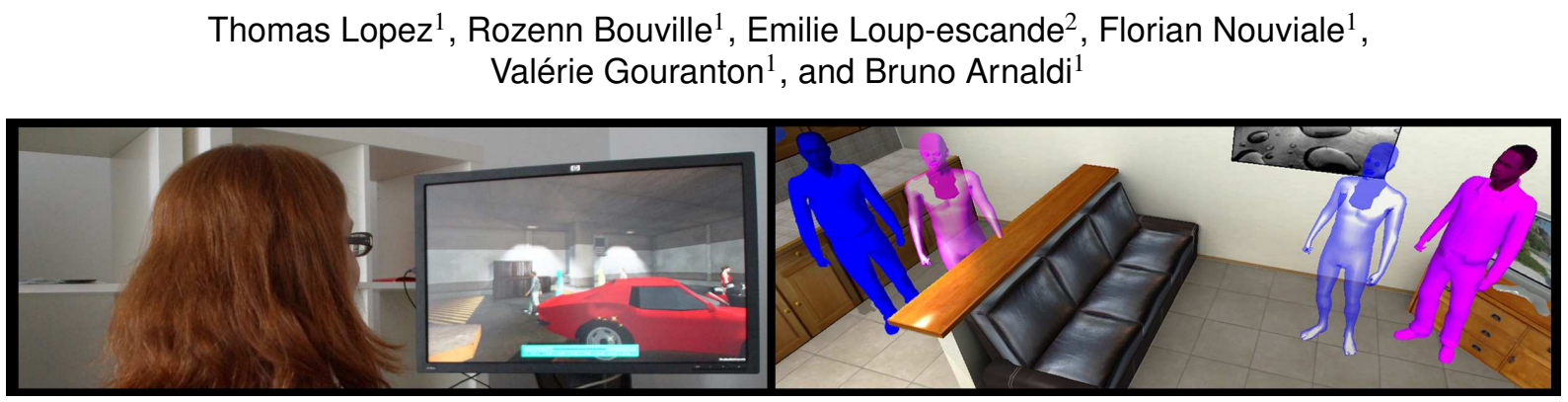

\begin{abstract}
The exchange of avatars, i.e. the actual fact of changing once avatar with another one, is a promising trend in multi-actor virtual environments. It provides new opportunities for users, such as controlling a different avatar for a specific action, retrieving knowledge belonging to a particular avatar, solving conflicts and deadlocks situations or even helping another user. Virtual Environments for Training are especially affected by this trend as a specific role derived from a scenario is usually assigned to a unique avatar. Despite the increasing use of avatar exchange, users' perception and understanding of this mechanism have not been studied. In this paper, we propose two complementary user-centered evaluations that aim at comparing several representations for the exchange of avatars; these are termed exchange metaphors. Our first experiment focuses on the perception of an exchange by a user who is not involved in the exchange, and the second experiment analyzes the perception of an exchange triggered by the user. Results show that the use of visual feedback globally aids better understanding of the exchange mechanism in both cases. Our first experiment suggests, however, that visual feedback is less efficient than a simple popup notification in terms of task duration. In addition, the second experiment shows that much simpler metaphors with no visual effect are generally preferred because of their efficiency.
\end{abstract}

Index Terms-Perception, User-centered evaluations, Virtual environments for training, Multi-user virtual environments

\section{INTRODUCTION}

Multi-actor virtual reality applications aim at immersing users in virtual environments populated with other actors, represented by avatars. Such virtual environments allow them to perform various actions and interactions that may be impossible in the real world. One of the most desired actions is perhaps taking control over another avatar, changing its incarnation on the fly to see the world through someone else's eyes. This takeover could have a wide range of motives: making another actor perform a specific action, accessing some knowledge possessed only by a particular avatar, resolving conflicts and deadlock situations or assisting another actor. The main difficulty in multi-actor environments is that avatars can be controlled by either real users or virtual agents. The takeover may then imply an exchange of control of the owners of the involved avatars. Moreover, in some environments, each avatar is also associated with specific metadata such as its own knowledge on the world and on the entities therein. Therefore, metadata coherence also needs to be taken into account during exchanges of avatars. For instance, in Virtual Environments for Training (VET), knowledge gathered by an avatar must remain available to its new owner after an exchange.

In the literature, recent contributions have described exchange protocols. To our knowledge, however, no user-centered evaluation focuses on the perception and understanding of the exchange of avatars. Following this idea, our work attempts to assess the key elements in the representation of an exchange between two actors' avatars, either real users or virtual agents, given one of its aims is to be understood by the users. In this study, we focus on multi-actor virtual environments where the user is embodied in an avatar with a first-person view. In the context of this paper, we are only interested in the visual represen-

- ${ }^{1}$ INSA of Rennes / IRISA / INRIA. E-mail: firstName.lastName[at]irisa.fr. - ${ }^{2}$ Ergonomics advisor for INRIA, Rennes tation of the exchange. Nevertheless, many visual parameters remain available to represent the same exchange of avatar, such as color, animation, duration, etc. In this paper, we refer to a specific configuration of these parameters as an exchange metaphor.

This study tries to analyze the key elements in the design of an exchange metaphor. We hypothesize that whether the user is involved in the exchange or just witnessing it a different metaphor should be used. Therefore, we designed two experiments to compare several exchange metaphors. In the first experiment, users witness exchanges, whereas in the second they are directly involved in the exchange. We also think that exchange metaphors should be tailored to the user's familiarity with virtual reality applications. We also explore the effect of abstract vs. explicit metaphors, as well as static vs. dynamic representations. We present here the results of these two experiments and highlight relevant elements that impact the design of an exchange metaphor for a virtual reality application. Results notably show that the use of visual feedback globally helps better understanding of the exchange mechanism. Visual feedback, however, can be less efficient than a simple popup notification in terms of task duration. Moreover, the use of simpler metaphors with no visual effect is generally preferred in triggering an exchange because of their efficiency.

The rest of the paper is organized as follows. First, the following Section 2 presents related work on role exchanges. In Section 3, we explain the concept of the exchange metaphor and the experiment's motivations. In Section 4 we describe our two experiments and give an analysis of the obtained results. Section 5 concludes.

\section{Related Work}

The role of an actor in a virtual environment has been defined as a coherent set of standard behaviors [4]. Role exchange, also termed role switching or role swapping, has been studied for many years in the Intelligent Robots field $[8,10]$ where it is used to improve the achievement of collaborative tasks. Indeed, the possibility of switching the role assigned to robots between leader and follower is an efficient way 
to speed up a task and to resolve conflicts. In the Virtual Reality domain, Evrard et al. [3] also take advantage of the possibility of switching between a leader and a follower role to improve the collaboration of users involved in a dyad haptic interaction for a physical collaborative task. More recently, role exchanges have also taken place in the context of human computer haptic collaboration [11]. The system provides haptic feedback to the user through a force negotiation mechanism, in order to help him/her achieve a dynamic task. Furthermore, the system is able to detect if the user needs help and, if necessary, switches from an assistive role to an equal-control role to complete the task.

The role notion is also very common in areas such as Interactive Storytelling [7] or Collaborative Virtual Environments for Training (CVET) $[2,14,20]$, notably to define interactions between autonomous agents. In virtual environments, the notion of role exchange and exchange of avatars are often mixed as each avatar possesses its own capabilities and thus exchanging roles includes exchanging of avatars. Role exchange is a particularly interesting feature for CVET. Indeed, Seers et al. [17, 18] studied team-member relationships and showed that the quality of lateral (within-group) relationships is as important as that of vertical relationships (with hierarchical supervisors) and even observed gains in production efficiency in self-managing teams. Therefore, the chance to learn the different roles associated with a procedure and to understand the work as a whole can reinforce the cohesion of the team. Thus, it is important for a trainee to be able to exchange his/her role with one of his/her teammates during training, so that he/she can better overview the collaborative procedure as a whole and understand more than a simple sequence of individual actions. In fact, role exchange proposes new usages for both designers and users for CVET and is also a promising and challenging trend for this area. It generates new concepts; for instance, in [16], an entity called the Perceptive Puppet and an interaction model [15] are described to allow trainees to exchange their avatars on the fly without interrupting the training procedure. Other CVETs also propose after-action review tools using role exchange to enhance experiences between users and virtual humans such as that used by Raij et al. [13]. Using the IPSViz tool, they offer the trainee the chance to take the role of an avatar that was previously his/her conversation partner. This is done during the review process so that the user can see how his/her performance was perceived through someone else's eyes and improve his/her behavior later on.

Moreover, various perception studies have been interested in the link between a user and the avatar he/she controls in the virtual world [9]. Such studies have been interested in disrupting the spatial unity between the self and the body [6] or in triggering the illusion that an artificial body was the user's one [12]. In these studies, multisensory information is manipulated to make users believe they are embodied in a new body, or avatar, and to analyze for instance their feeling of ownership toward their new body. Slater et al. [19] conducted a perception study about the experience of body transfer in a virtual environment. In this study, the user point of view was modified during the experimentation allowing the user to watch the same scene sequentially from different perspectives, from an external viewpoint to a view embodied in the main character's eyes. This study aimed at analyzing differences in the users reactions depending on their point of view, in other word their role, in the virtual environment. The exchange metaphor used in this experimentation was not analyzed, however.

The exchange of avatars or roles is a useful tool for virtual reality applications, CVET and perception studies. This concept is also used in video games when a user has to control different characters, such as in the last Grand Theft Auto $5^{1}$ where the player can swap character to enhance his/her game experience. Despite the increasing interest in exchanges of avatars, however, no user-centered evaluation has yet focused on the perception and understanding of this mechanism.

${ }^{1}$ http://www.rockstargames.com/V/

\section{TOWARD THE EVALUATION OF EXCHANGE METAPHORS}

\subsection{Motivations}

The exchange of avatars gives the user the possibility of taking control over another avatar to see and act in the environment from a brand new perspective. This exchange can result from different reasons: control of multiple avatars at the same time, making an avatar perform a specific action, or accessing some knowledge it possesses but also resolving conflicts and deadlock situations or assisting another actor. For instance, in some video games, this exchange is used so that the player can control one of many characters and change at any time. Moreover, in the context of VET, the exchange allows a trainer to switch his/her role with a trainee so that he/she can show a particular manipulation or gesture, for instance in a surgery procedure.

This exchange brings new challenges to multi-user virtual environments. First, users must comprehend they are involved in an exchange process. Second, they must understand they are now embodied in a new avatar. Third, they must be able to locate themselves in the virtual environment as the exchange might disorient them. Moreover, they also need to deal with various metadata associated with their new avatars such as new capabilities, different knowledge on the environment or on simulation history and so on. Although exchanges of avatar have often been used in the literature $[13,16,19]$, the method of representing this exchange has never been evaluated.

This study proposes to tackle this issue by proposing a first evaluation of different exchange metaphors. The term Exchange Metaphor refers to the set of parameters defining such a representation of an exchange of avatars in a virtual environment. This metaphor can use various multisensory information such as sounds or visual effects to represent the exchange. In this study, we only focus on the visual representation of these metaphors. Still, a multitude of parameters are available to define a metaphor as a combination of visual elements : color, animation, speed, duration, text display, camera effects, etc. As a study covering the set of all possible parameters is impossible to conduct, this paper focuses on exchange metaphors using an intentionally limited set of these parameters.

\subsection{Design of the metaphor evaluation}

The purpose of this study is more to determine the relevant criteria involved in designing exchange metaphors for a given application than to highlight the best metaphor. In order to design our experiments, we distinguished two groups of exchange metaphors as follows:

- The user witnesses the exchange. In this case, the user needs to perceive and comprehend an exchange of avatars between other actors in the environment. For instance, a trainee is learning a procedure which requires the help of his/her trainer while this trainer is exchanging his/her avatar with another actor in the environment. Therefore, the trainee needs to understand the exchanges in order to ask for his/her assistance. Our first evaluation focuses on comparing different metaphors with this witness condition of the user.

- The user triggers the exchange of his/her own avatar. Here, the user needs to be able to easily trigger his/her exchange with another avatar and acknowledge that the exchange is being performed. Unlike the previous experiment, the participant is now involved in his/her own exchange. For example, the user is watching a surgical procedure and wants to pursue the surgical procedure by exchanging his/her avatar with the current surgeon. $\mathrm{He} / \mathrm{she}$ then asks for control of the surgeon's avatar and processes to the exchange of avatars. Therefore, our second evaluation compares several metaphors designed specifically for this experimental condition.

These two groups are complementary as they target two different conditions of exchange of avatars, depending on whether the user is involved or not. We do not study the case where users are involved in an exchange they did not trigger. We consider that, in this situation, a notification would appear to inform the user that another actor is 
asking for an exchange of avatar. If the user then accepts the request, the exchange should be triggered, which is equivalent to our second experiment.

Despite the need to separate our analysis into two experiments, we want to analyze common criteria for both conditions. These criteria can be categorized in two groups: Understanding and Perception of the metaphor. Therefore, we used for this analysis several variables that fall within the user experience. This user experience is defined as a consequence of an interaction between a user (with his/her characteristics) and a product (with its features and qualities) after an evaluation process [5]. The criteria we use to measure this user experience for the different exchange metaphors are defined as follows:

- Understanding: the user should easily and quickly understand what is happening and who is involved in the exchange. To evaluate comprehension, we use criteria such as the ease of understanding, the users' subjective feelings, the number of errors and the duration of sessions.

- Perception: the exchange metaphor should be pleasant to use. Moreover, it should not be disturbing for the user as this could dissuade him/her from using it. It is evaluated with criteria such as the aesthetic aspect, the originality of the metaphor, or the degree of excitement and pleasant assessed by the user.

\section{Evaluation of the exchange metaphors}

We present two complementary experiments defined as follows. Our first experiment compares three exchange metaphors. Here, participants are not involved in the exchange but are only witnesses of several exchanges of avatars. Our second experiment compares five exchange metaphors. Unlike the first experiment, participants are now involved in the exchange as they trigger the exchange of their own avatar. The purpose of these two experiments is to analyze the perception and the comprehension of the exchange of both a spectator and an actor in the exchange.

In this section we present the two experimental protocols we used as well as the results we obtained. The first experiment is presented in Section 4.2 and the second in Section 4.3. For each experiment, Subsection (1) presents the goal and the design of the metaphors. Then, we detail the experimental groups (2), the procedure (3) and the collected data (4). The results we obtained are explained in Subsections (5). Through these two experiments we noted some interesting differences but also some commonalities between the novice and the expert users which are detailed in Subsections (6).

\subsection{Experimental Setup}

Experiment 1 took approximately 30 minutes to complete and experiment 2 took approximately 20 minutes. Participants were encouraged to take breaks between each trial to avoid fatigue. Participants came from various educational backgrounds and were all unaware of the purpose of the experiments. We also balanced the groups to get a similar number of male and female participants. They were mainly recruited from a university and administration background.

Participants used an application designed with the engine Unity 4 and RocketBox Studios characters. The experiment for all of the participants ran on a 30 inch monitor with a resolution of $1920 \times 1200$ pixels. Participants were not able to see the different experimental conditions beforehand. Moreover, the experiments were conducted in a room without environmental noise or any other source of distraction.

\subsection{Experiment 1: The User Witnesses the Exchange}

\subsubsection{Objective and Study Design}

This first experiment compares several exchange metaphors in order to evaluate which one is more usable or provides a more positive experience to a user who witnesses an exchange. To do this, we decided to compare different metaphors using static visual feedback, animated visual feedback or simple 2D-GUI. Therefore, we designed three metaphors: (1) an on-site visual metaphor, (2) a moving and animated visual metaphor and (3) a 2D-GUI with a text and a static image. These representations used a color code; each virtual owner of an avatar was represented by a different color in the metaphor. The exchange metaphors are defined as follows:

1. Flickering avatar $\left(M_{A}(\circledast)\right)$ : the two avatar textures blink several times from white to the color of their new owner (cf. Fig.1(a)).

2. Ghost translation $\left(M_{B}(\nexists)\right)$ : a ghost appears besides each of the two original avatars and moves to the location of the newly possessed avatar. Each ghost is colored according to the owner's color representation (cf. Fig.1(b)). These ghosts materialize owners in a visual transition representing their disembodiments from their former avatar and their embodiments in the new one.

3. Popup notification $\left(M_{C}(\square)\right)$ : For each of the two avatars involved in an exchange, a textbox displays the picture of the avatar, with a background color matching the new owner's color and a label specifying the new owner's name (cf. Fig.1(c)).

To avoid introducing a confounding factor from variable exposure durations, all the metaphors have the same display duration. Moreover, as we wanted to assess if the participants were aware of the exchanges of avatar in the environment, no information was displayed apart from the metaphors to inform the participant of who was controlling which avatars (A demonstration video of these three metaphors is presented here : http://youtu.be/Yu4YpgzKXes ). Table 1 sums up these different representations. Moreover, a glimpse of these metaphors is presented in Fig. 1 which shows the same exchange with the three metaphors used in this experiment.

Table 1. Metaphors Compared in Experiment 1.

\begin{tabular}{|c|c|c|}
\hline Metaphor name & Visual representation & Symbol \\
\hline Metaphor A & Flickering avatar & $M_{A}(\circledast)$ \\
\hline Metaphor B & Ghost translation & $M_{B}(\nexists)$ \\
\hline Metaphor C & Popup Notification & $M_{C}(\square)$ \\
\hline
\end{tabular}

\subsubsection{Participants}

Fifty-four participants ( 7 females and 47 males, aged from 18 to 63, mean=28.6, sd=7.8) took part in the experiment. The experiment was divided into three experimental groups, one for each of our three metaphors. Each participant was assigned to one of these experimental groups according to four criteria: gender, age, occupation and knowledge in virtual reality (VR). The experimental groups are balanced as homogeneous as possible using those four criteria and consisted of 18 participants. The groups were characterized in Table 2.

\subsubsection{Procedure}

Each participant was first informed about the complete study proceedings. Then, they completed an identification questionnaire allowing us to collect general information about their demographics, occupation and experience with VR. Next, a demonstration video introduced the exchange metaphor associated with their experimental group and gave instructions about the objective. The objective consisted in performing a scenario composed of nine tasks in order to repair a vehicle in the environment presented in Fig. 2. Participants had to perform the tasks presented in Table 3. For each task, participants had to go to a specified area of the environment and then to act on the designated elements. The scenario alternated between individual $(I)$ and collaborative tasks $(C)$ which were displayed one after the other during the session. These tasks did not require any a priori knowledge on the vehicle or on mechanics and were explained to the participants one after the other during each session.

During the experiment, participants viewed three avatars whose purpose was to assist the user during collaborative tasks. Participants were informed that each avatar was controlled by an agent who was 


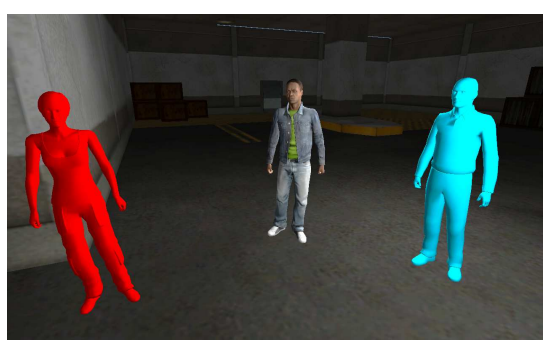

(a) Flickering avatar

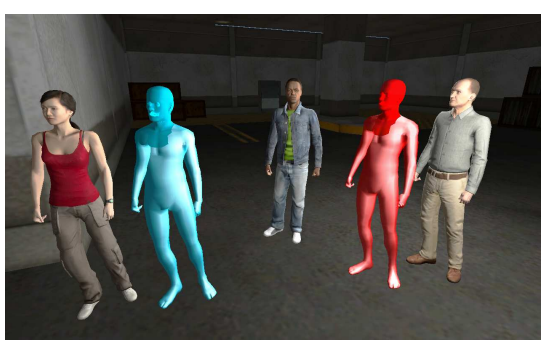

(b) Ghost translation

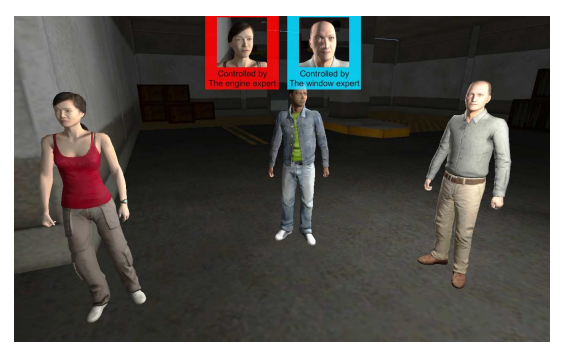

(c) Popup notification

Fig. 1. Presentation of the metaphors used in experiment 1: the blue expert is exchanging its avatar with the red expert, taking control of the right avatar.

Table 2. Distribution of the Participants among our Three Experimental Groups for Experiment 1.

\begin{tabular}{|c|c|c|c|c|}
\hline \multicolumn{2}{|c|}{ Criteria } & $M_{A}(\circledast)$ & $M_{B}(\nexists)$ & $M_{C}(\square)$ \\
\hline \multirow{2}{*}{ Gender } & Man & 15 & 16 & 16 \\
& Woman & 3 & 2 & 2 \\
\hline \multirow{5}{*}{ Age } & $15-25$ & 5 & 6 & 7 \\
& $25-35$ & 10 & 10 & 10 \\
& $35-45$ & 2 & 2 & 0 \\
& $>45$ & 1 & 0 & 1 \\
\hline \multirow{5}{*}{ Occupation } & Administration & 2 & 1 & 0 \\
& Technician & 0 & 1 & 0 \\
& PhD Student & 3 & 2 & 4 \\
& Other Student & 5 & 5 & 5 \\
& Post-doctoral & 3 & 2 & 1 \\
& Engineer & 4 & 6 & 7 \\
& Lecturer & 1 & 1 & 1 \\
\hline \multirow{5}{*}{ VR } & None & 1 & 2 & 2 \\
& Minimal & 4 & 4 & 4 \\
& Some & 6 & 4 & 5 \\
& Good & 7 & 8 & 7 \\
\hline
\end{tabular}

Table 3. Tasks Composing the Scenario the Participants had to Perform. A Task is either Individual (I) or Collaborative (C).

\begin{tabular}{|c|c|}
\hline Task & Required Expert \\
\hline Recharge the battery (I) & - \\
\hline Replace the engine (C) & Engine Expert \\
\hline Check the tire pressure (I) & - \\
\hline Replace a front wheel (C) & Wheel Expert \\
\hline Change the rear light bulb (I) & - \\
\hline Replace the windscreen (C) & Window Expert \\
\hline Repair the front bumper (I) & - \\
\hline Add oil to the engine (C) & Engine Expert \\
\hline Clean the windows (I) & - \\
\hline
\end{tabular}

an expert in a specific area (a wheel expert, a window expert and an engine expert) and that they must ask the appropriate expert for help with each collaborative task. For example, the wheel expert was required to change a wheel. Each expert was also associated with a specific color: yellow (wheel expert), blue (window expert) and red (engine expert). These colors were the same across all experimental conditions so that participants could recognize which experts were exchanging their avatars. We also presented participants at the beginning of the experiment with the avatars and their controlling experts (avatar A is controlled by the wheel expert,...). During each individual task in the scenario, exchanges of avatars were performed between the three experts. All the exchanges were scripted to ensure similar conditions

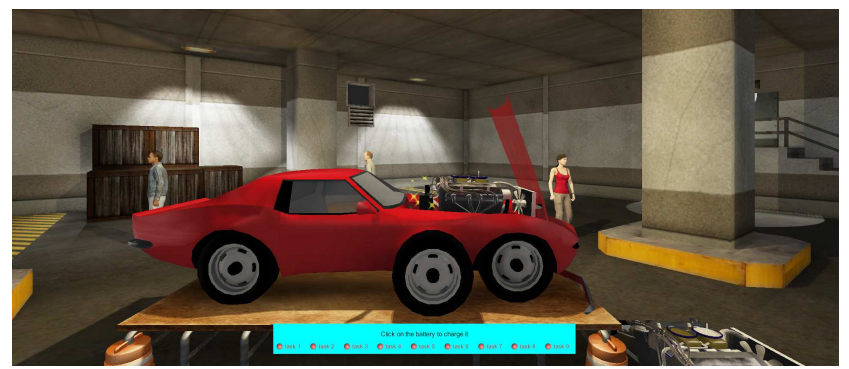

Fig. 2. Environment used in experiment 1.

for all the participants. During these exchanges participants were able to continue the task they were doing and were not forced to watch the whole exchange protocol. All participants performed the same scenario three times, with different expert exchanges for each trial. After each trial, participants had to indicate in which avatar each of the three experts was embodied. After the third and final trial, participants were asked to fill in a questionnaire to provide their impressions, comments and subjective judgments. They were not asked to perform the scenario as quickly as possible; we expected, however, to observe some learning effect on the procedure completion time regardless of the metaphor used.

\subsubsection{Collected Data}

For each trial and each participant, we recorded the total duration of the trials as well as the number of errors (i.e., a wrong expert has been selected). At the end of the experiment, the participants had to fill in a subjective questionnaire with [A] a Likert-scale (1: not agree at all, 7: totally agree) for the following criteria: (1) the metaphor is easy to understand, (2) the metaphor is easy to use, (3) the metaphor distracts you from the task and (4) this metaphor is a good idea; and [B] a semantic differential: this exchange metaphor is (5) strange versus familiar, (6) obstructing versus supporting, (7) ordinary versus original, (8) boring versus exciting, (9) unpleasant versus pleasant and (10) unaesthetic versus aesthetic Open-ended questions concerning positive and negative aspects as well as suggestions to improve the metaphor where also added in the questionnaire. The significant results obtained for this experiment are summed up in Table 6 and detailed in Section 4.2.5.

\subsubsection{Results}

In this experiment, each participant was testing only one metaphor. Therefore, we used the independent-sample measurement techniques for the statistical analysis.

When the Test of Normality (i.e., Shapiro Wilk test) indicated a significant result $(p<0.05)$, suggesting a violation of the assumption of normality, we used Kruskal-Wallis and Mann-Whitney tests. Sig- 


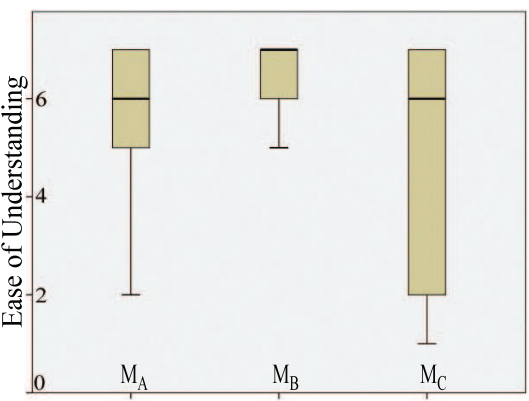

(a) Ease of understanding

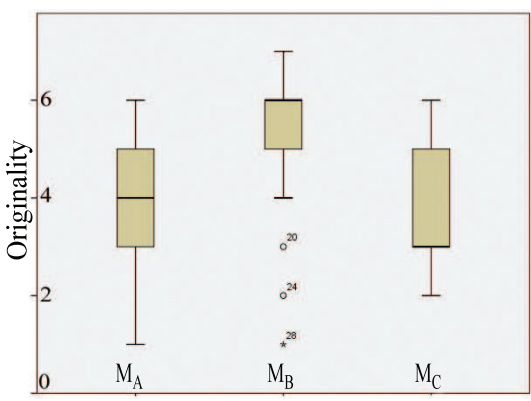

(d) Originality

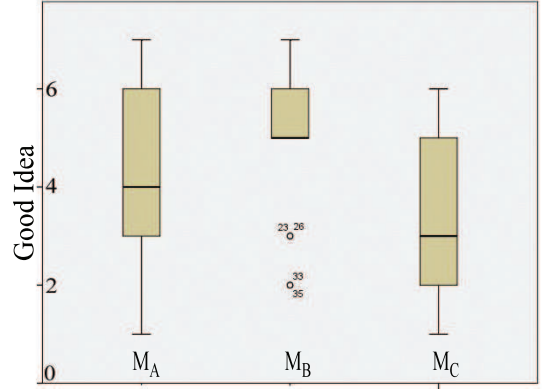

(b) Good idea

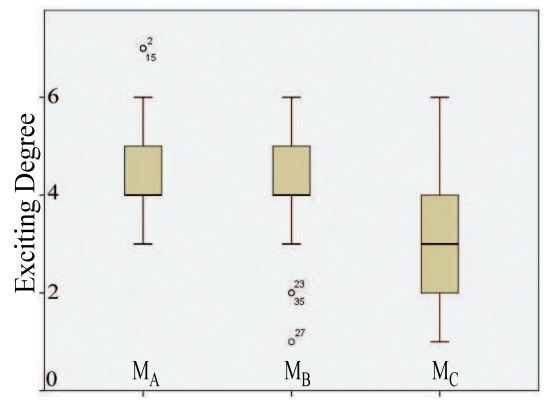

(e) Excitement

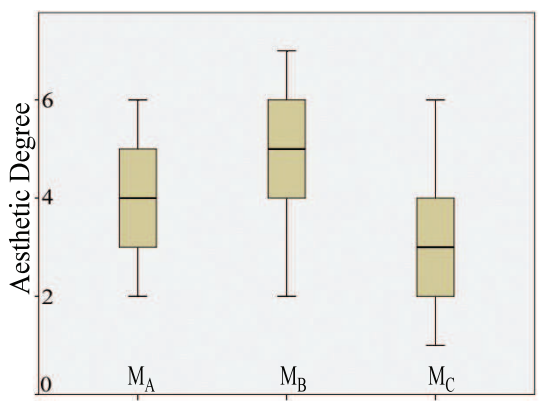

(c) Aesthetic

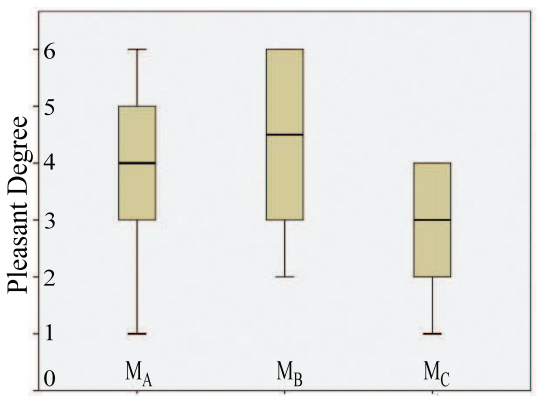

(f) Pleasant

Fig. 3. Boxplots of the marks attributed to each metaphor in experiment 1.

nificant differences between the three metaphors were found for six criteria: ease of understanding, good idea, originality of the metaphor, exciting degree, pleasant degree and task duration. The computed significant differences are presented in Table 4. No significant difference was computed for the remaining criteria using this test. The significant differences between the pairs of metaphors using Mann-Whitney tests for the different criteria are summed up in Table 5. Whenever a significant difference is observed in our results, please refer to this table to find the associated values. When the Test of Normality (i.e., Shapiro Wilk test) indicated a non-significant difference $(p>0.05)$, suggesting a validation of the assumption of normality, we used OneWay Between-Groups ANOVA and Tukey HSD tests. Thus, with regard to the aesthetic degree, we performed an ANOVA analysis of variance that showed a statistically significant difference between the metaphors $(F(2,51)=3.78, p<0.05)$.

Most of our results are presented in boxplots (see Fig.3). These boxplots are delimited by the quartiles (25\%- and $75 \%$-quartile) of the distribution of the condition over all the individuals. Moreover, the medians, minima and maxima are given for each boxplot. Each significant result is explained in a paragraph where the first bold sentence presents the conclusion and the relevant tests are detailed after.

Table 4. Significant Differences Computed with the Kruskal-Wallis Test.

\begin{tabular}{|c|c|c|}
\hline Variable & \multicolumn{2}{|c|}{ Kruskal-Wallis test } \\
\hline & $\chi^{2}(2, n=54)$ & $p$ \\
\hline Ease of understanding & 8.61 & $<0.05$ \\
\hline Good idea & 7.31 & $<0.05$ \\
\hline Originality & 9.87 & $<0.05$ \\
\hline Exciting degree & 9.23 & $<0.05$ \\
\hline Pleasant degree & 8.37 & $<0.05$ \\
\hline Task duration & 9.59 & $<0.05$ \\
\hline
\end{tabular}

Metaphor $M_{B}(\nexists)$ is easier to understand, perceived as a better idea and more aesthetic than metaphor $M_{C}(\square)$. The Mann-Whitney
Table 5. Significant Differences Computed with the Mann-Whitney Test Between Two Metaphors $(p<0.05)$.

\begin{tabular}{|c|c|c|}
\hline Metaphor Comparison & $U$ & $p$ \\
\hline \multicolumn{3}{|c|}{ Criterion : Ease of understanding } \\
\hline$M_{B}(\nexists)$ vs $M_{C}(\square)$ & 78 & $<0.05$ \\
\hline \multicolumn{3}{|c|}{ Criterion : Good idea } \\
\hline$M_{B}(\nexists)$ vs $M_{C}(\square)$ & 82.50 & $<0.05$ \\
\hline \multicolumn{3}{|c|}{ Criterion: Originality } \\
\hline$M_{A}(\circledast)$ vs $M_{B}(\nexists)$ & 91.50 & $<0.05$ \\
\hline$M_{B}(\nexists)$ vs $M_{C}(\square)$ & 70.50 & $<0.005$ \\
\hline \multicolumn{3}{|c|}{ Criterion : Excitement degree } \\
\hline$M_{A}(\circledast)$ vs $M_{C}(\square)$ & 74.50 & $<0.005$ \\
\hline$M_{B}(\nexists)$ vs $M_{C}(\square)$ & 92.50 & $<0.05$ \\
\hline \multicolumn{3}{|c|}{ Criterion : Pleasant degree } \\
\hline$M_{A}(\circledast)$ vs $M_{C}(\square)$ & 91.50 & $<0.05$ \\
\hline$M_{B}(\nexists)$ vs $M_{C}(\square)$ & 83.50 & $<0.05$ \\
\hline \multicolumn{3}{|c|}{ Criterion : Task duration } \\
\hline$M_{A}(\circledast)$ vs $M_{C}(\square)$ & 69 & $<0.005$ \\
\hline$M_{B}(\nexists)$ vs $M_{C}(\square)$ & 92.50 & $<0.05$ \\
\hline
\end{tabular}

test indicated a significant difference only between $M_{B}(\nexists)$ and $M_{C}(\square)$, which suggests that metaphor $M_{B}(\nexists)$ is easier to understand than metaphor $M_{C}(\square)$ (see Fig.3(a)). In the same vein, this test also suggests that metaphor $M_{B}(\nexists)$ is globally a better idea than metaphor $M_{C}(\square)$ (see Fig.3(b)). Concerning the aesthetic degree, post-hoc comparisons using the Tukey HSD test indicated that metaphor $M_{B}(\nexists)$ was perceived as more aesthetic than metaphor $M_{C}(\square)$ (see Fig.3(c)). $M_{A}(\circledast)$ showed no significant difference from either $M_{B}(\nexists)$ or $M_{C}(\square)$.

Metaphor $M_{B}(\nexists)$ is more original than the others. The MannWhitney test indicated significant differences between $M_{A}(\circledast)$ and $M_{B}(\nexists)$, and between $M_{B}(\nexists)$ and $M_{C}(\square)$. This suggests that metaphor $M_{B}(\nexists)$ was judged more original than both $M_{A}(\circledast)$ and $M_{C}(\square)$ (see Fig.3(d)). 
Table 6. Categorization of the Relevance of each Metaphor by Significant Differences (+ rated Significantly Higher, - rated Significantly Lower, 0 no Significant Difference).

\begin{tabular}{cccccccc}
\hline & Ease of understanding & Good idea & Aesthetic & Originality & Excitement degree & Pleasant & Task duration \\
\hline$M_{A}(\circledast)$ & 0 & 0 & 0 & - & + & + & - \\
$M_{B}(\nexists)$ & + & + & + & + & + & + & - \\
$M_{C}(\square)$ & - & - & - & - & - & - & + \\
\hline
\end{tabular}

Metaphor $M_{C}(\square)$ is more boring and unpleasant than the others. With the Mann-Whitney test, significant differences were observed between $M_{A}(\circledast)$ and $M_{C}(\square)$ concerning the boring and pleasant degrees as well as between $M_{B}(\nexists)$ and $M_{C}(\square)$. This suggests that metaphor $M_{C}(\square)$ was judged more boring than the metaphors $M_{A}(\circledast)$ and $M_{B}(\nexists)$ (see Fig.3(e)) but also less pleasant (see Fig.3(f)).

The group using $M_{C}(\square)$ was faster than the other groups. In this case, a Mann-Whitney test indicated significant difference between $M_{A}(\circledast)$ and $M_{C}(\square)$ as well as between $M_{B}(\nexists)$ and $M_{C}(\square)$. This suggests that the mean duration of the task accomplishment with metaphor $M_{C}(\square)(214 \mathrm{sec})$ was shorter than with $M_{A}(\circledast)(256 \mathrm{sec})$ and $M_{B}(\nexists)(247 \mathrm{sec})$ (see Fig.4).

No difference in terms of ease of use, distraction, facility, obstruction and number of errors was found between these metaphors. This suggests that no metaphor is better than another regarding these criteria.

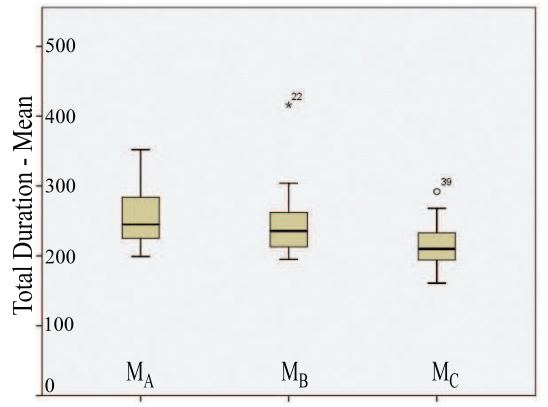

Fig. 4. Boxplots of the "duration of the task accomplishment" for each metaphor.

\subsubsection{Novice versus Expert Users}

Table 7. Task Duration (in seconds) by User Expertise: Novice Users are Faster than Expert Users with $M_{C}(\square)$.

\begin{tabular}{|c|c|c|}
\hline Metaphor & Expert Mean & Novice Mean \\
\hline$M_{A}(\circledast)$ & 235 & 270 \\
\hline$M_{B}(\nexists)$ & 240 & 252 \\
\hline$M_{C}(\square)$ & 228 & 205 \\
\hline
\end{tabular}

For this experiment, we had a total of 32 novice users and 22 expert users. We did not find any significant differences between novice and expert ratings for ease of understanding, excitement, and pleasant and aesthetic degrees. Whatever the metaphor, however, the exchange metaphors were considered a better idea by the novice users (mean= 4.53 ) than by the expert users (mean $=3.72)$. Finally, the most interesting difference between these two groups concerns the duration of the task. We globally observed that there was a similar learning effect for all the users, as the completion task improved along the three trials. It is interesting to note, however, that the mean completion time for

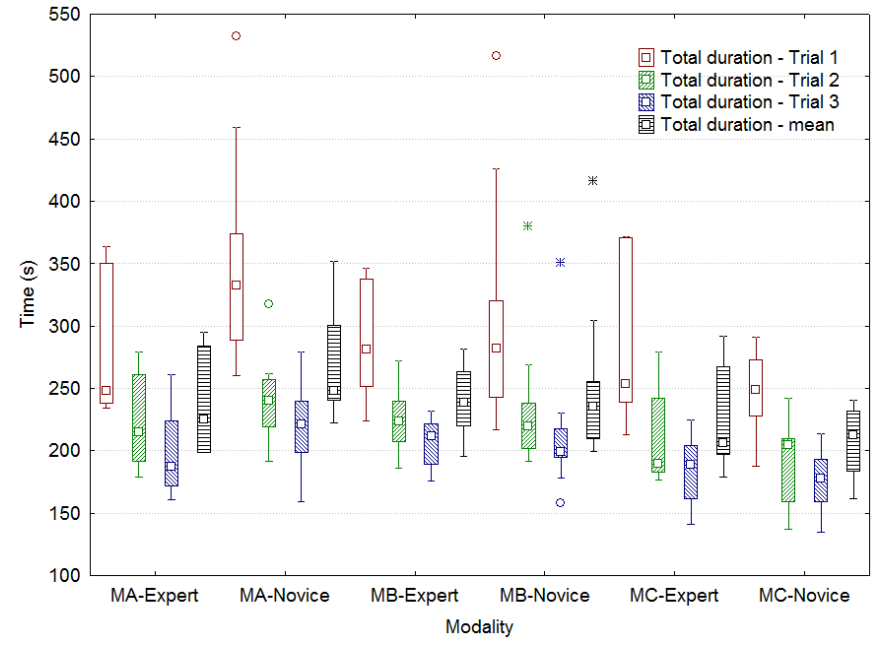

Fig. 5. Task duration for the novice and expert users : boxplots of the three trials and mean time.

the expert users was globally the same whatever the metaphor, unlike the case with novice users (see Table 7). We can see that using visual metaphors $M_{A}(\circledast)$ and $M_{B}(\nexists)$ makes the task harder for novice users as they spend more time completing it. Yet it is particularly interesting that not only the use of a popup notification helps the novice users to achieve their task faster ( mean $=205 \mathrm{sec}$ ), but also that, using this metaphor, the novice users are even faster than the expert users. This difference is not statistically significant $(p>0.05)$, however.

\subsubsection{Conclusion}

The significant differences on the various variables of this experiment are summed up in Table 6. To conclude, it appears that metaphor $M_{B}(\nexists)$ was perceived by participants as being the easiest to understand, the best idea, the most original and the most aesthetic. Conversely, metaphor $M_{C}(\square)$ was perceived as the most unpleasant and boring. This metaphor $M_{C}(\square)$ was the fastest one regarding the session duration, however. Thus, depending on the purpose of the application, the metaphor has to be different. On the one hand, for a userfriendly application, a visual metaphor such as $M_{B}(\nexists)$ would be more suitable. On the other hand, for an application requiring a fast task accomplishment, a pop-up notification in the user interface would be more efficient. Whatever the choice, it should have no consequences for either ease of use or the number of errors made. Finally, it would also be interesting to combine the two metaphors $M_{B}(\nexists)$ and $M_{C}(\square)$ in order to test if this combination improve or, on the contrary, creates an important workload for the user that complicates his/her work and understanding. The better perception of visual feedback by the participants as witnesses of the exchanges led us to conduct the second experiment to check if the use of visual feedback also helps when the participants trigger their own exchange. 


\subsection{Experiment 2: The User Triggers the Exchange of His/Her Own Avatar}

\subsubsection{Objective and Study Design}

This second experiment aims at comparing five exchange metaphors to study, if and why one is preferred and provides a more positive user experience. Differently from the previous experiment, participants were now involved in their exchanges as they triggered them.

In this experiment, the exchange metaphors were designed with two parameters : the camera motion and the visual feedback. We used two camera motions : a straight linear motion between the two avatars $(\rightarrow)$ and an external point of view combined with a camera motion $(\curvearrowright)$. The straight linear motion followed a direct camera trajectory from the participant avatar's perspective to that of his/her new avatar. The external point of view (external-PoV) motion started and ended at the same positions as the previous motion but pauses at an intermediate point, showing both avatars involved in the exchange. The second parameter concerns the visual feedback. We designed metaphors with visual feedback (noted $\boldsymbol{\nu}^{\prime}$ ) and others without visual feedback (noted $\emptyset)$. Metaphors with visual effects used the same feedback defined in the first experiment (Ghost translations + Flickering avatars) in order to provide additional information about the exchange. Combining the camera parameter $(\rightarrow / \curvearrowright)$ and the visual feedback parameter $(\nu /$ $\emptyset)$ gave us four different metaphors : $M_{1}\left(\rightarrow, \nu^{\prime}\right), M_{2}(\rightarrow, \emptyset), M_{3}\left(\curvearrowright, \nu^{\prime}\right)$ and $M_{4}(\curvearrowright, \emptyset)$. In addition to these four metaphors, we added another, $M_{5}(\emptyset, \emptyset)$, which used neither camera motions nor visual feedback: the camera was instantly set to the new avatar's perspective. We assumed that this fifth metaphor defined the simplest representation for taking control over a new avatar. Table 8 gives the definitions of these five metaphors. Figure 6 presents a user's view of this experiment. A demonstration video of these metaphors is presented here : http: //youtu.be/Yu4YpGzKXes.

Table 8. Metaphors Compared in Experiment 2.

\begin{tabular}{|c|c|c|c|}
\hline Metaphor & Camera motion & Visual effect & Symbol \\
\hline$M_{1}$ & linear $(\rightarrow)$ & with $(\boldsymbol{\prime})$ & $M_{1}(\rightarrow, \boldsymbol{\prime})$ \\
\hline$M_{2}$ & linear $(\rightarrow)$ & without $(\emptyset)$ & $M_{2}(\rightarrow, \emptyset)$ \\
\hline$M_{3}$ & external-PoV $(\curvearrowright)$ & with $\left(\boldsymbol{\nu}^{\prime}\right)$ & $M_{3}\left(\curvearrowright, \boldsymbol{\nu}^{\prime}\right)$ \\
\hline$M_{4}$ & external-PoV $(\curvearrowright)$ & without $(\emptyset)$ & $M_{4}(\curvearrowright, \emptyset)$ \\
\hline$M_{5}$ & none $(\emptyset)$ & without $(\emptyset)$ & $M_{5}(\emptyset, \emptyset)$ \\
\hline
\end{tabular}

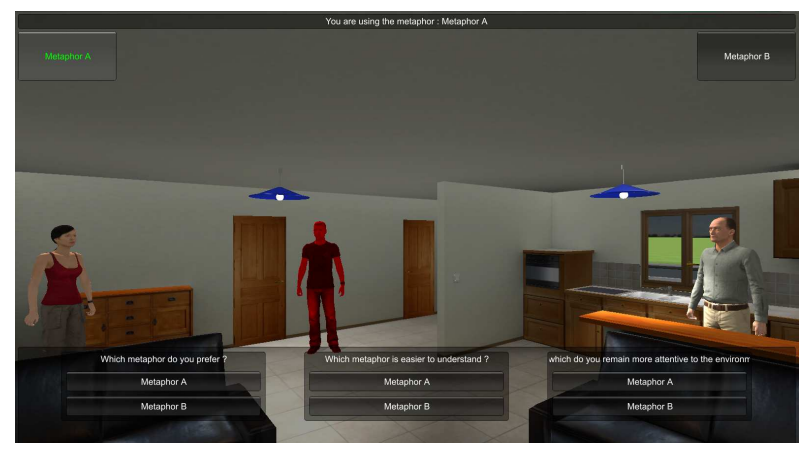

Fig. 6. Presentation of the environment of the second experimentation: the use can trigger the exchange of his own avatar with any of the three other avatars in the environment.

\subsubsection{Participants}

Forty-two participants ( 3 females and 39 males, aged from 18 to 63, mean=29.2, sd=9.3) took part in this experiment. They were distributed as presented in Table 9 .
Table 9. Distribution of the Participants by Criteria for Experiment 2.

\begin{tabular}{|c|c||c|c||c|c|}
\hline Age & $\mathrm{N}(/ 42)$ & Occupation & $\mathrm{N}(/ 42)$ & VR Skills & $\mathrm{N}(/ 42)$ \\
\hline & & PhD Student & 11 & & \\
$15-25$ & 16 & Other Student & 12 & None & 3 \\
$25-35$ & 21 & Post-doctoral & 2 & Minimal & 10 \\
$35-45$ & 1 & Engineer & 12 & Some & 11 \\
$>45$ & 4 & Lecturer & 3 & Good & 18 \\
& & Researcher & 2 & & \\
\hline
\end{tabular}

\subsubsection{Procedure}

Each participant was first informed about the complete test proceedings. Then they were asked to complete an identification questionnaire allowing us to collect general information about their demographics, occupation and experience with VR. After that, a demonstration video introduced the five exchange metaphors to them and gave instructions about the task they had to complete. The participants were asked to compare each of the five metaphors two by two which led to a total of ten comparisons. These ten comparisons were presented in a different order for each participant to avoid any undesirable learning effect. The participants completed different questionnaires. First, a questionnaire was filled in after each comparison to collect their preferences between the two compared metaphors. Then, at the end of the experiment, they filled in a final questionnaire to provide more global impressions, comments and subjective judgments.

\subsubsection{Collected Data}

We first counted the number of times each metaphor was preferred to another. In addition, at the end of the experiment, participants had to fill in a subjective questionnaire on each of the five metaphors with [A] a Likert scale (1: not agree at all, 7: totally agree) for the following criteria: (1) this metaphor is easy to understand, (2) this metaphor is a good idea; and $[\mathrm{B}]$ a semantic differential such as: this exchange metaphor is (3) Ordinary versus Original, (4) Boring versus Excitement, and (5) Unpleasant versus Pleasant. Open-ended questions concerning positive and negative aspects as well as suggestions to improve the metaphor where also added in the questionnaire. Finally, at the end of the questionnaire, participants were asked to rank the five metaphors in order of preference and to indicate the metaphor they would retain if they had to design a virtual environment with an exchange of avatars. The significant results obtained are summed up in Table 12 and detailed in the Section 4.3.5.

\subsubsection{Results}

In this experiment, participants had to test five exchange metaphors. Therefore, we used paired-sample measurement techniques for the statistical analysis. A Test of Normality (i.e., Shapiro Wilk) indicated a significant result $(p<0.05)$ suggesting a violation of the assumption of normality for all the tested variables, i.e. the population of participants was never normally distributed for the considered variables. For this reason, we used Friedman and Wilcoxon Signed Rank tests for numerical variables (e.g., mark assigned by the participants to the item "Good idea": scores ranging from 1 to 7) while we used the Chi-Square test for independence for categorical variables (e.g., rank of metaphor: first, second, third, fourth and fifth). In order to facilitate the reading of the results, all Friedman test results showing significant differences are presented in Table 11. Table 10 sums up all of the computed Wilcoxon test values for the different criteria. Most of our results are presented in boxplots (see Fig. 8). These boxplots are delimited by the quartiles (25\%- and $75 \%$-quartile) of the distribution of the condition over all the individuals. Moreover, the medians, minima and maxima are represented in each boxplot. Each significant result is explained in a small paragraph where the first bold sentence presents the conclusion and the rest of the paragraph details the tests we used.

Metaphors $M_{1}(\rightarrow, \nu), M_{2}(\rightarrow, \emptyset)$ and $M_{5}(\emptyset, \emptyset)$ were globally preferred. Concerning the participants' preferred metaphors, Wilcoxon 
Table 10. Results Computed with Wilcoxon's Test: White Cells Indicate a Significant Difference Between the Two Variables $(p<0.05)$ in Contrast to Gray Cells.

\begin{tabular}{|c|c|c|c|c|c|c|c|c|}
\hline Metaphor & \multicolumn{2}{|c|}{$M_{1}\left(\rightarrow, \nu^{\prime \prime}\right)$} & \multicolumn{2}{|c|}{$M_{2}(\rightarrow, \emptyset)$} & \multicolumn{2}{|c|}{$M_{3}\left(\curvearrowright, \nu^{\prime \prime}\right)$} & \multicolumn{2}{|c|}{$M_{4}(\curvearrowright, \emptyset)$} \\
\hline & $z$ & $p$ & $z$ & $p$ & $z$ & $p$ & $z$ & $p$ \\
\hline \multicolumn{9}{|c|}{ Criterion : Preferred Metaphors } \\
\hline$M_{3}\left(\curvearrowright \nu^{\prime \prime}\right)$ & -3.25 & $<0.005$ & -3.06 & $<0.005$ & & & & \\
\hline$M_{4}(\curvearrowright, \emptyset)$ & -4.38 & $<5.10^{-4}$ & -4.83 & $<0.005$ & -3.01 & $<0.005$ & & \\
\hline$M_{5}(\emptyset, \emptyset)$ & & $>0.05$ & -3.34 & 0.005 & -2.13 & $<0.05$ & -3.68 & $<5.10^{-}$ \\
\hline \multicolumn{9}{|c|}{ Criterion : Kept Metaphor } \\
\hline$M_{4}(\curvearrowright, \emptyset)$ & -2.31 & $<0.05$ & -2.11 & $<0.05$ & & $>0.05$ & & \\
\hline$M_{5}(\emptyset, \emptyset)$ & & $>0.05$ & & $>0.05$ & & $>0.05$ & -3.15 & $<0.005$ \\
\hline \multicolumn{9}{|c|}{ Criterion : Ease of understanding } \\
\hline $\bar{M}(\rightarrow, \emptyset)$ & -2.83 & $<0.005$ & & & & & & \\
\hline$M_{4}(\curvearrowright, \emptyset)$ & -5.01 & $<5.10^{-4}$ & -3.55 & $<5.10^{-4}$ & -4.34 & $<5.10^{-2}$ & & \\
\hline$M_{5}(\emptyset, \emptyset)$ & -3.95 & $<5.10^{-4}$ & -2.13 & $<0.05$ & -2.39 & $<0.05$ & & $>0.05$ \\
\hline \multicolumn{9}{|c|}{ Criterion : Excitement degree } \\
\hline$M_{4}(\curvearrowright, \emptyset)$ & -3.50 & $<5.10^{-4}$ & -2.93 & $<0.005$ & -2.47 & $<0.05$ & & \\
\hline$M_{5}(\emptyset, \emptyset)$ & & $>0.05$ & & $>0.05$ & & $>0.05$ & -2.90 & $<0.005$ \\
\hline \multicolumn{9}{|c|}{ Criterion : Pleasant degree } \\
\hline$M_{4}(\curvearrowright, \emptyset)$ & -2.29 & $<0.05$ & -3.70 & $<5.10^{-4}$ & -2.86 & $<0.005$ & & \\
\hline$M_{5}(\emptyset, \emptyset)$ & & $>0.05$ & & $>0.05$ & & $>0.05$ & -3.34 & $<0.005$ \\
\hline \multicolumn{9}{|c|}{ Criterion : Good Idea } \\
\hline$M_{2}(\rightarrow, \emptyset)$ & & $>0.05$ & & & & & & \\
\hline$M_{3}\left(\curvearrowright, \nu^{\prime \prime}\right)$ & & $>0.05$ & & $>0.05$ & & & & \\
\hline$M_{4}(\curvearrowright, \emptyset)$ & -3.55 & $<5.10^{-4}$ & -3.77 & $<5.10^{-4}$ & -4.14 & $<5.10^{-}$ & & \\
\hline$M_{5}(\emptyset, \emptyset)$ & & $>0.05$ & & $>0.05$ & & $>0.05$ & -3.13 & 0.005 \\
\hline \multicolumn{9}{|c|}{ Criterion: Original } \\
\hline$M_{2}(\rightarrow, \emptyset)$ & -3.48 & 0.005 & & & & & & \\
\hline$M_{3}\left(\curvearrowright, \nu^{\prime}\right)$ & -3.73 & $5.10^{-4}$ & -4.96 & $5.10^{-4}$ & & & & \\
\hline$M_{4}(\curvearrowright, \emptyset)$ & & $>0.05$ & -3.83 & $5.10^{-4}$ & -3.73 & $5.10^{-4}$ & & \\
\hline$M_{5}(\emptyset, \emptyset)$ & -3.88 & $5.10^{-4}$ & -2.80 & 0.005 & -5.03 & $5.10^{-4}$ & -4.53 & $5.10^{-}$ \\
\hline
\end{tabular}

Table 11. Significant Differences Computed with the Friedman's Test for Each Variable.

\begin{tabular}{|c|c|c|}
\hline Variable & \multicolumn{2}{|c|}{ Friedman values } \\
\hline & $\chi^{2}(4, n=42)$ & $p$ \\
\hline "Preferred metaphor" & 44.24 & $<5.10^{-4}$ \\
\hline "Kept metaphor" & 11.10 & $<0.05$ \\
\hline Ease of understanding & 45.43 & $<5.10^{-4}$ \\
\hline Excitement degree & 16.42 & $<0.005$ \\
\hline Pleasant degree & 16.62 & $<0.005$ \\
\hline Good idea & 22.17 & $<5.10^{-4}$ \\
\hline Originality & 68.138 & $<5.10^{-4}$ \\
\hline
\end{tabular}

tests indicated significant differences between $M_{3}\left(\curvearrowright, \nu^{\prime}\right)$ and the other four metaphors $\left(M_{1}\left(\rightarrow, \nu^{\prime}\right), M_{2}(\rightarrow, \emptyset), M_{4}(\curvearrowright, \emptyset)\right.$ and $\left.M_{5}(\emptyset, \emptyset)\right)$ and also between $M_{4}(\curvearrowright, \emptyset)$ and the four other metaphors.

Comparing the number of times each metaphor was preferred by participants, we found that $M_{3}\left(\curvearrowright, \nu^{\prime}\right)$ and $M_{4}(\curvearrowright, \emptyset)$ were less popular than metaphors $M_{1}\left(\rightarrow,,^{\prime}\right), M_{2}(\rightarrow, \emptyset)$ and $M_{5}(\emptyset, \emptyset)$. The resulting histogram is presented in Figure 7(a). Moreover, the fact that metaphor $M_{4}(\curvearrowright, \emptyset)$ was the least favorite is also confirmed by the question "If you had to design an environment needing an exchange metaphor, which exchange metaphor would you keep?" with a Wilcoxon test that showed significant differences between $M_{4}(\curvearrowright, \emptyset)$ and metaphors $M_{1}(\rightarrow, \nu), M_{2}(\rightarrow, \emptyset)$ and $M_{5}(\emptyset, \emptyset)$. This suggests participants would retain metaphors $M_{5}(\emptyset, \emptyset), M_{1}(\rightarrow, \nu)$ and $M_{2}(\rightarrow, \emptyset)$ and would not keep the metaphor $M_{4}(\curvearrowright, \emptyset)$ (see Fig.7(b) for values).

The preference for metaphors $M_{1}(\rightarrow, \downarrow), M_{2}(\rightarrow, \emptyset)$ and $M_{5}(\emptyset, \emptyset)$, and the rejection of $M_{3}(\curvearrowright, \nu)$ and $M_{4}(\curvearrowright, \emptyset)$ were also corroborated by the final ranking performed by the participants. Indeed, a Chi-
Square test for independence indicated a significant association between metaphor and rank $\left(\chi^{2}(16, n=210)=91.76, p<0.0005\right)$. Figure $7(\mathrm{c})$ shows that metaphor $M_{5}(\emptyset, \emptyset)$ was mainly ranked first, that $M_{2}(\rightarrow, \emptyset)$ was mainly ranked second and that $M_{1}(\rightarrow, \nu)$ was mainly ranked second and third. Metaphors $M_{3}\left(\curvearrowright, \nu^{\prime}\right)$ and $M_{4}(\curvearrowright, \emptyset)$ were respectively mainly ranked fourth and fifth.

Metaphors $M_{1}\left(\rightarrow, \nu^{\prime}\right), M_{2}(\rightarrow, \emptyset)$ and $M_{3}\left(\curvearrowright, \nu^{\prime}\right)$ were easier to understand than $M_{4}(\curvearrowright, \emptyset)$ and $M_{5}(\emptyset, \emptyset)$. Concerning the ease of understanding, a Wilcoxon test indicated significant differences between:

- $M_{1}\left(\rightarrow, \boldsymbol{\nu}^{\prime}\right)$ and metaphors $M_{2}(\rightarrow, \emptyset), M_{4}(\curvearrowright, \emptyset), M_{5}(\emptyset, \emptyset)$;

- $M_{2}(\rightarrow, \emptyset)$ and metaphors $M_{1}\left(\rightarrow, \boldsymbol{\nu}^{\prime}\right), M_{4}(\curvearrowright, \emptyset), M_{5}(\emptyset, \emptyset)$;

- $M_{3}\left(\curvearrowright, \nu^{\prime}\right)$ and metaphors $M_{4}(\curvearrowright, \emptyset), M_{5}(\emptyset, \emptyset)$.

Regarding Figure 8(a), this suggests that metaphors $M_{1}\left(\rightarrow, \boldsymbol{\nu}^{\prime}\right)$, $M_{3}\left(\curvearrowright, \nu^{\prime}\right)$ and also $M_{2}(\rightarrow, \emptyset)$ were perceived as easier to understand than metaphors $M_{4}(\curvearrowright, \emptyset)$ and $M_{5}(\emptyset, \emptyset)$.

Metaphor $M_{4}(\curvearrowright, \emptyset)$ is more boring, unpleasant and a bad idea compared with the other metaphors. Wilcoxon test indicated significant differences between $M_{4}(\curvearrowright, \emptyset)$ and the other four metaphors (for details, see Table 10) suggesting that metaphor $M_{4}(\curvearrowright, \emptyset)$ was less liked by the participants. Figure 8 presents the resulting boxplots for the experiment on the excitement degree (Fig.8(c)), the pleasant degree (Fig.8(d)) and whether the metaphor was a good idea (Fig.8(b)).

Metaphors $M_{3}\left(\curvearrowright, \nu^{\prime}\right), M_{1}\left(\rightarrow, \nu^{\prime}\right)$ and $M_{4}(\curvearrowright, \emptyset)$ are more original than metaphors $M_{2}(\rightarrow, \emptyset)$ and $M_{5}(\emptyset, \emptyset)$. Concerning originality, a Wilcoxon test indicated significant differences between $M_{3}\left(\curvearrowright, \nu^{\prime}\right), M_{2}(\rightarrow, \emptyset)$ and $M_{5}(\emptyset, \emptyset)$ from the four other metaphors. Regarding Figure 8(e), this suggests that $M_{3}\left(\curvearrowright, \nu^{\prime}\right), M_{1}\left(\rightarrow, \nu^{\prime}\right)$ and $M_{4}(\curvearrowright$ $, \emptyset)$ were perceived as more original than $M_{2}(\rightarrow, \emptyset)$ and $M_{5}(\emptyset, \emptyset)$ but also that metaphor $M_{3}(\curvearrowright, \nu)$ was considered as the most original one. 


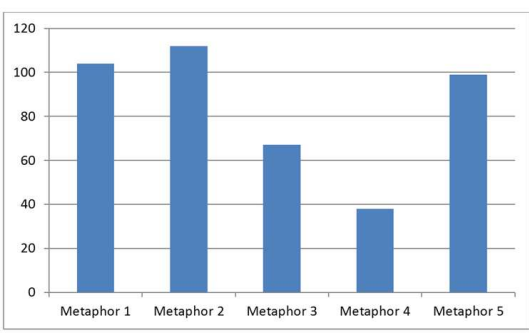

(a) Number of times each metaphor has been preferred for all of the comparisons.

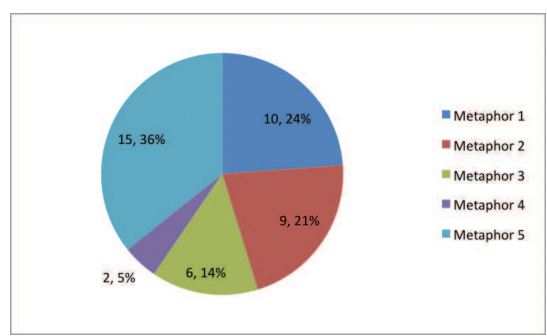

(b) Metaphor finally selected by participants to represent the exchange

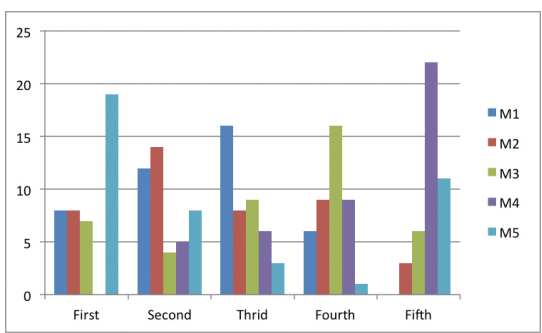

(c) Ranking of the metaphor from first to fifth.

Fig. 7. The metaphors $M_{1}\left(\rightarrow, \boldsymbol{\nu}^{\prime}\right), M_{2}(\rightarrow, \emptyset)$ and $M_{5}(\emptyset, \emptyset)$ were globally preferred to $M_{3}\left(\curvearrowright, \boldsymbol{\nu}^{\prime}\right)$ and $M_{4}(\curvearrowright, \emptyset)$.

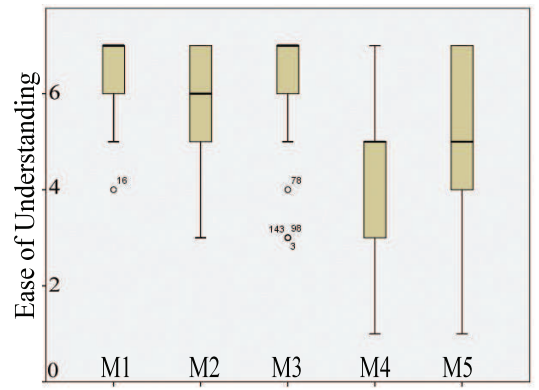

(a) Ease of understanding

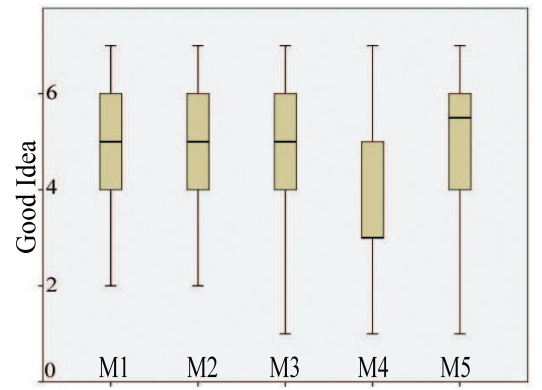

(b) Good idea

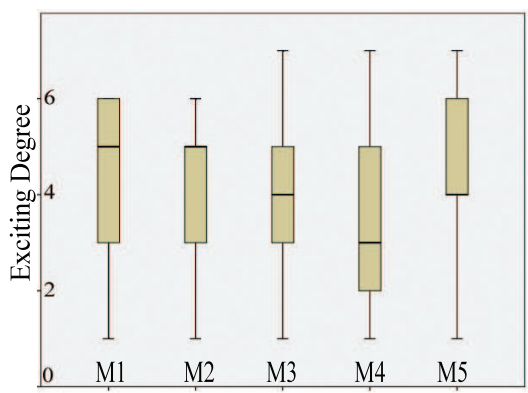

(c) Excitement

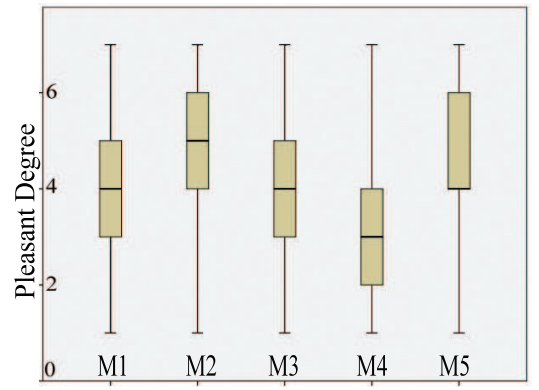

(d) Pleasant

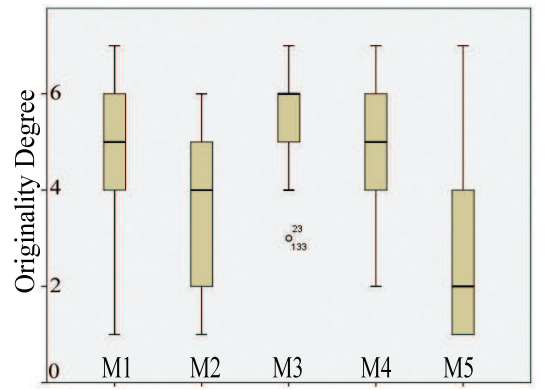

(e) Originality

Fig. 8. Boxplots of the marks attributed to each metaphor for experiment 2.

\subsubsection{Novice versus Expert Users}

In this experiment, we employed 18 novice and 24 expert users. The novice users showed significant results $(p<0.05)$ for two criteria: originality and ease of understanding. On these criteria, novice and expert users globally had the same significant differences regarding the metaphors. They notably agreed on the originality of the metaphors, i.e. $M_{3}\left(\curvearrowright, \nu^{\prime}\right)$ was very original and $M_{5}(\emptyset, \emptyset)$ very ordinary. Expert users also showed significant differences for ease of understanding, pleasant degree and whether the metaphor was a good idea or not. On these criteria, they strongly rejected $M_{4}(\curvearrowright, \emptyset)$. Regarding users comments, this was caused by the "loss of reference points" during the "perturbing camera motions" and because $M_{4}(\curvearrowright, \emptyset)$ was "too slow".

\subsubsection{Conclusion}

Table 12 sums up the significant differences observed on the variables. To conclude, the use of the linear motion $(\rightarrow)$ of the camera is preferred to the use of the curved motion $(\curvearrowright)$. Indeed, regarding the participants' comments, the immersion feeling is better with the linear motion as users "feel" they are moving from one avatar to the other. Moreover, the use of visual feedback helps users to understand the ex- change, as it strengthens the impression of several users in the same environment. Regarding the comments of the participants, these visual effects also helped them to understand that they were not "moving" in the environment but "exchanging" avatars. Some participants felt, however, that the visual feedback distracted them (i.e. some explained that the vivid colors and ghosts monopolized their attention, causing a lack of focus on what was happening around). This distraction explains why the same number of participants chose to keep $M_{1}(\rightarrow, \boldsymbol{\nu})$ or $M_{2}(\rightarrow, \emptyset)$. Finally, the simplest metaphor, $M_{5}(\emptyset, \emptyset)$, was the one the mostly ranked first by users. This can be explained by its "execution speed", as underlined by the comments of several participants but also because it was judged as ordinary by the participants (see Fig. 8(e)) who might have been more familiar with its use. This metaphor was judged hard to understand and some participants suggested a learning phase using a metaphor such as $M_{1}(\rightarrow, \downarrow)$ for better understanding of the exchange before the use of $M_{5}(\emptyset, \emptyset)$ for the efficiency of the real application. Not surprisingly, these results are in accordance with studies on travel in virtual environments [1], as exchange could indeed be seen as a particular case of navigation in a virtual environment. 
Table 12. Categorization of the Relevance of Each Metaphor by Significant Difference (+ Rated Significantly Higher, - Rated Significantly Lower, 0 No Significant Difference).

\begin{tabular}{ccccccc}
\hline & Preferred & Easier to understand & Excitement & Pleasant & Good idea & More original \\
\hline$M_{1}\left(\rightarrow, \nu^{\prime \prime}\right)$ & + & ++ & + & + & + & + \\
$M_{2}(\rightarrow, \emptyset)$ & + & + & + & + & + & - \\
$M_{3}\left(\curvearrowright, \nu^{\prime \prime}\right)$ & - & + & + & + & + & ++ \\
$M_{4}(\curvearrowright, \emptyset)$ & - & - & - & - & - & + \\
$M_{5}(\emptyset, \emptyset)$ & + & - & + & + & + & - \\
\hline
\end{tabular}

\section{CONCLUSION}

The exchange of avatars opens new perspectives for multi-actor virtual applications, notably in the field of virtual environments for training where several studies reveal that the exchange of avatars is an efficient way to improve the training $[13,16]$. To our knowledge, however, no study has focused on how to represent an exchange of avatar efficiently. To that end, we proposed two complementary experiments that aim at assessing the crucial parameters that make the representation of an exchange, called an exchange metaphor, more comprehensible to and better perceived by the user. The first experiment focused on exchanges witnessed by the user, whereas the second experiment, focused on exchanges triggered by the user for his/her own avatar. These experiments enable us to offer several recommendations for the design of comprehensible and well-perceived exchange metaphors.

First, the experiments confirmed that different parameters should be taken into account to define the exchange metaphor depending on whether the user is involved or not in the exchange. In both cases, however, the use of visual effects generally helps to explain the exchange mechanism but could distract the user from the task.

Second, several metaphors can be proposed depending on the level of familiarity of the end-user with VR. Comprehensible and familiar metaphors should be proposed to novice users whereas expert users will prefer efficient metaphors. Moreover, a learning phase should be available for novice users to allow them to use more efficient metaphors once they are used to the exchange mechanism.

Finally, metaphors must be adapted to the targeted application: user-friendly metaphors, despite being well-appreciated by users, often lead to a longer task accomplishment time, especially for novice users, even though no difference has been found in terms of either ease of use or exchange awareness. Further, we claim that the metaphor must be adapted to the context of the exchange. An example of such a context-dependent exchange metaphor, based on the distance, is present in the video game Grand Theft Auto 5. Here, a metaphor close to $M_{5}(\emptyset, \emptyset)$ is used when avatars are next to each other and a metaphor using the principle of $M_{4}(\curvearrowright, \emptyset)$ is used when characters are distant.

We have considered several ways to extend this work. We could, for instance, combine visual feedback with other stimuli, like auditory feedback, in order to improve the exchange metaphors. Finally, we would like to extend our experiment as we strongly believe that depending on conditions the metaphors should also be different. For instance, we could investigate whether the present results would be confirmed in more complex and larger virtual environments. Moreover, we intend to examine the influence of the user's immersion degree or the distance between the avatars involved in the exchange. Regarding the immersion degree of the application, we believe that when the user triggers the exchange, a direct forward camera motion should be preferred to preserve the immersion feeling rather than an instantaneous viewpoint change that could introduce disorientation and sickness.

\section{ACKNOWLEDGMENTS}

This work was supported by the French Research National Agency project named CORVETTE (ANR-10-CONTINT-CORD-012) and by the French Unique Interdepartmental Funds SIFORAS (FUI 11).

\section{REFERENCES}

[1] D. Bowman, D. Koller, and L. Hodges. Travel in immersive virtual environments: an evaluation of viewpoint motion control techniques. In Virtual Reality Annual International Symposium, IEEE, pp 45-52, 1997.

[2] J. Dugdale, B. Pavard, N. Pallamin, M. el Jed, and C. L. Maugan. Emergency fire incident training in a virtual world. In Proceedings $I S$ CRAM2004, volume 167, 2004.

[3] P. Evrard and A. Kheddar. Homotopy switching model for dyad haptic interaction in physical collaborative tasks. In EuroHaptics conference and Symposium on Haptic Interfaces for Virtual Environment and Teleoperator Systems, pages 45-50, 2009.

[4] A. Guye-Vuilleme and D. Thalmann. A high-level architecture for believable social agents. Virtual Reality, 5(2):95-106, 2000.

[5] M. Hassenzahl and N. Tractinsky. User experience-a research agenda. Behaviour \& Information Technology, 25(2):91-97, 2006.

[6] B. Lenggenhager, T. Tadi, T. Metzinger, and O. Blanke. Video ergo sum: manipulating bodily self-consciousness. Science, 317:1096-1099, 2007.

[7] I. Machado, A. Paiva, and R. Prada. Is the wolf angry or... just hungry? In Proceedings of the fifth international conference on Autonomous agents, pages 370-376. ACM, 2001.

[8] E. Martinson and R. Arkin. Learning to role-switch in multi-robot systems. In IEEE International Conference on Robotics and Automation, 2003. Proceedings. ICRA 03, pages 2727-2734 vol.2, 2003.

[9] A. Maselli and M. Slater. The building blocks of the full body ownership illusion. Front Hum Neurosci, 7, 032013.

[10] E. Monacelli, C. Riman, R. Thieffry, I. Mougharbel, and S. Delaplace. A reactive assistive role switching for interaction management in cooperative tasks. In 2006 IEEE/RSJ International Conference on Intelligent Robots and Systems, pages 5118-5123, 2006.

[11] S. Oguz, A. Kucukyilmaz, T. Sezgin, and C. Basdogan. Haptic negotiation and role exchange for collaboration in virtual environments. In 2010 IEEE Haptics Symposium, pages 371-378, 2010.

[12] V. I. Petkova and H. H. Ehrsson. If i were you: perceptual illusion of body swapping. PloS one, 3(12):e3832, 2008.

[13] A. B. Raij and B. C. Lok. Ipsviz: An after-action review tool for humanvirtual human experiences. In Virtual Reality Conference, 2008. VR'08. IEEE, pages 91-98. IEEE, 2008.

[14] J. Rickel and W. L. Johnson. Virtual humans for team training in virtual reality. In Proceedings of the ninth international conference on artificial intelligence in education, volume 578, page 585. Citeseer, 1999.

[15] A. Saraos Luna, V. Gouranton, and B. Arnaldi. Collaborative Virtual Environments For Training: A Unified Interaction Model For Real Humans And Virtual Humans. In Edutainment, pages 1-12, 2012.

[16] A. Saraos Luna, V. Gouranton, B. Arnaldi, et al. The perceptive puppet: Seamless embodiment exchange between real and virtual humans in virtual environments for training. In International Conference on Computer Graphics Theory and Applications, pages 1-6, 2013.

[17] A. Seers. Team-member exchange quality: A new construct for rolemaking research. Organizational behavior and human decision processes, 43(1):118-135, 1989.

[18] A. Seers, M. M. Petty, and J. F. Cashman. Team-member exchange under team and traditional management: A naturally occurring quasiexperiment. Group and Organization Management, 20(1):18-38, 1995.

[19] M. Slater, B. Spanlang, M. V. Sanchez-Vives, and O. Blanke. First person experience of body transfer in virtual reality. PloS one, 5(5), 2010.

[20] K. Sycara and G. Sukthankar. Literature review of teamwork models. Technical report, Robotics Institute, 2006. 